\title{
Las Familias ante las Escuelas en Estudios Chilenos: Algunos Supuestos Bajo Sospecha
}

\section{Families in front of the Schools in Chilean Researches: Some Suspicious Assumptions}

\author{
Dominique Manghi ${ }^{1}$, Elsa María Castrillón-Correa ${ }^{2, *}$, Héctor Cárcamo-Vásquez ${ }^{3}$ y Andrea Precht ${ }^{4}$ \\ ${ }^{1}$ Pontificia Universidad Católica de Valparaíso, Chile \\ ${ }^{2}$ Universidad Católica del Maule, Chile \\ ${ }^{3}$ Universidad del Bío-Bío, Chile \\ ${ }^{4}$ Centro de Investigación para la Educación en Justicia Social (CIEJUS), Chile
}

\section{DESCRIPTORES:}

Familia; Escuela; Relación padresescuela; Relación escuela-comunidad, Fondos de conocimiento.

\section{RESUMEN:}

El trabajo problematiza la producción de sentidos respecto de las relaciones entre las familias y las escuelas en Chile. Analizamos 25 artículos correspondientes a la producción científica chilena de 9 autores desde iniciados los años 2000. El análisis utiliza como marco interpretativo el enfoque Fondos de Conocimiento y, es orientado a partir de los siguientes interrogantes: ¿quiénes son los agentes involucrados?, ¿por qué se relacionan?, ¿para quélo hacen? y ¿cómo se expresa dicha relación? Como hallazgos destacan, primero, la forma de configurar la noción de familia y de escuela, en donde se aprecia un desbalance entre la precisión con la que se define escuela y la imprecisión respecto de familia y, segundo, las maneras de acudir a ella, al identificar que la relación opera desde una lógica instrumental. Los estudios refieren a familia en términos generales, asumiendo consenso en su definición. Se concluye que la investigación como quehacer social tiene un efecto performativo contribuyendo a la naturalización de la familia desde el principio de funcionalidad/disfuncionalidad. Los resultados invitan a levantar la sospecha sobre la noción de familia cristalizada en las investigaciones y a abrirse a la experiencia subjetiva de los sujetos y prevenir definiciones normativas y tradicionales.

\section{KEYWORDS:}

Family; School; Parentschool relationship; School-community relationship, Funds of knowledge.

\section{ABSTRACT:}

This paper discusses the production of meaning concerning relationships between families and schools in Chile. We analyse 25 articles corresponding to the Chilean scientific production of 9 authors since the beginning of the 2000s. The analysis uses the Funds of Knowledge approach as an interpretive framework and is guided by the following questions: who are the agents involved? why do they relate to each other? what is their purpose?, and how is this relationship expressed? Findings highlight how the concept of family is configured and how it is used. Regarding the first, we see an imbalance between the precision with which the school is defined and imprecision regarding the family. Concerning the second, we highlight an instrumental logic in the relationship. The studies refer to the family in general terms, assuming consensus in its definition. It is concluded that research as a social task has a performative effect, contributing to the naturalization of the family under the principle of functionality/dysfunctionality. The results invite us to raise suspicions about the notion of family crystallized in research and to open up to the subjective experience of the subjects and to avoid normative and traditional definitions.

CÓMO CITAR:

Manghi, D., Castrillón-Correa, E. M., Cárcamo-Vásquez, H. y Precht, A. (2021). Las familias ante las escuelas en estudios chilenos: Algunos supuestos bajo sospecha. Revista Internacional de Educación para la Justicia Social, 10(1), 27-40.

https://doi.org/10.15366/riejs2021.10.1.002

*Contacto: elsa castrillon@alu.ucm.cl

ISSN: 2254-3139

revistas.uam.es/riejs
Recibido: $\quad 5$ de octubre 2020

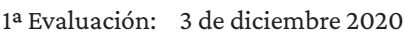

2a Evaluación: 19 de marzo 2021

Aceptado: $\quad 6$ de abril 2021 


\section{Introducción}

La relación entre familias y escuelas ha sido abordada latamente en los estudios científicos. La diversidad de enfoques teóricos utilizados para su abordaje, de posicionamientos disciplinares desde donde se generan los estudios y de diseños metodológicos adoptados en las investigaciones, permiten reconocer lo complejo y multifacético de esta interacción. En este sentido, diversas dimensiones parecen constituir la relación entre familias y escuelas.

Lejos de ser relaciones naturales, los lugares y funciones tanto de escuelas como familias en la educación responden a supuestos generalmente no problematizados. La investigación no está aislada de las luchas entre distintas formas de producción de sentidos (Slee y Allan, 2001), estos se mueven por el circuito académico como una constelación de objetos cristalizados por discursos económicos y políticos que requieren de una mirada crítica para abrirse a otras opciones de ver la realidad en movimiento (Zemelman, 2005).

Tal es el caso de los Fondos de Conocimiento como un ejemplo en que la investigación especula e intenciona un sentido particular y ético para una relación entre escuelas y familias. Este surge en los años noventa en una zona fronteriza de EE.UU con gran presencia mexicana, como una iniciativa de investigadores en antropología y educación de la Universidad de Arizona. Ellos se acercan a las escuelas para entregar principios orientadores que modifiquen la mirada deficitaria respecto de las familias de sus estudiantes (Esteban-Guitart et al., 2012). Proponen que los profesores sean aprendices y las familias sus profesores, pues poseerán un conocimiento valioso (Amanti, 2020). La mirada etnográfica permitió reconocer cuerpos de conocimientos, habilidades y estrategias acumulados históricamente y desarrollados culturalmente, los que se denominan Fondos de Conocimiento (Moll et al., 2001). Las bases teóricas provienen de un enfoque materialista de la cultura, de la psicología histórica-cultural (Moll, 2014) y de la teoría de redes sociales que reconoce que los estudiantes también dominan Fondos de Conocimiento que aprenden de abuelos, hermanos, tíos y primos con quienes mantienen relaciones cotidianas y "densas" (Vélez-Ibáñez y Greenberg, 2005).

En este artículo utilizaremos la nomenclatura "relaciones entre familias y escuelas", no solo porque pone en valor la pluralidad de modelos familiares y la diversidad de escuelas, sino también para superar la concepción monolítica que prevalece al discutir sobre la relación que se suscita entre dichas instituciones. Si bien la escuela tiene una matriz que sostiene su afán normalizador, como investigadores estimamos relevante-asumiendo dicha naturaleza- que es más pertinente hablar de tipos de relaciones entre familias y escuelas si queremos que nuestros modelos teóricos resuenen de forma más precisa (Díaz de Rada, 2010), en el actual contexto social y cultural. Pues no solo las familias y escuelas son diversas, sino también las relaciones que se establecen entre sus miembros.

Dichas relaciones no ocurren en abstracto, en nuestro caso, se sitúan en la sociedad neoliberal chilena y un modo de ser escuela que favorece la naturalización de dicho modelo, con un panorama educativo enmarcado en un contexto político, económico y cultural conocido como el "laboratorio neoliberal" (Bellei et al., 2008). Inspirados en el enfoque de Fondos de Conocimiento (FDC), asumimos una actitud de sospecha, que permite problematizar las afirmaciones o poner en duda aquello que se presenta como obvio, natural o normal (Cerletti, 2008) en las consideraciones relacionadas con familias y escuelas y, nos preguntamos cómo es que la investigación de los últimos veinte años ha contribuido en la producción de sentidos respecto de las relaciones entre familias y escuelas en Chile.

El objetivo del artículo es problematizar la conceptualización cristalizada en las investigaciones que involucran a las escuelas y familias en tanto objeto de investigación. Se busca transparentar algunos puntos de partida respecto del constructo de familia y recuperar otras opciones como los FDC, que puedan aportar a superar el enfoque individualista que atraviesa la experiencia de familias y escuelas en el contexto neoliberal. Se considera un total de 25 estudios de 9 investigadores chilenos -incluidos aquellos de los propios autores-, en el periodo posterior a los años 2000. El criterio de selección de los artículos, obedece a que son investigaciones que abordan el campo temático de las relaciones que involucran familias y escuelas. 
A continuación, problematizamos la definición de familias desde las políticas educativas e investigaciones, atendiendo a la producción de sentidos respecto de las familias y sus relaciones con las escuelas: ¿Quiénes son? ¿Por qué se relacionan? ¿Para qué? ¿Cómo se expresa dicha relación?

\section{2. ¿Cómo están construidas las familias en las políticas educativas chilenas? Una contextualización}

La investigación en educación y las políticas públicas en el ámbito educativo se entrelazan en el tiempo y, en el caso de Chile, pareciera que mayormente la investigación surge a partir de los temas que propone la política local (Manghi et al., 2020). Las políticas educativas de los últimos cincuenta años son determinantes para comprender la educación, las escuelas y sus actores.

La dictadura cívico-militar implementó en los años ochenta una reforma educacional que trasladó el foco desde un Estado docente a uno subsidiario, mediante el traspaso de la administración de las escuelas públicas desde el Estado hacia los municipios y sostenedores privados, dando paso a la apertura de un cuasi mercado educacional (Bellei et al., 2008; Sisto, 2019). En este, las escuelas compiten por la preferencia de las familias, traducida en captación de matrícula que, en el caso de las subvencionadas, se materializa en un financiamiento estatal en correlación con la asistencia diaria de los alumnos (Bellei et al., 2008; Donoso-Díaz et al., 2016). La Libertad de Enseñanza naturaliza el derecho de los padres a elegir la educación de sus hijos, reducida a la elección de escuela con base en su capacidad de pago (Alarcón y Donoso, 2017). Finalmente, son las escuelas quienes seleccionan a las familias siguiendo criterios económicos.

Con la llegada de la democracia se mantuvo la misma estructura y un énfasis en la gestión, introduciendo la noción de calidad educativa y escuela eficaz (Alarcón y Donoso, 2017). La calidad se mide mediante una práctica cuestionable que promueve concepciones simplistas del cambio educativo, reduciendo la evaluación a la aplicación de test estandarizados (Angulo, 2019). Mientras, la Ley de Jornada Escolar Completa (JEC) del año 1997 reestructura los tiempos escolares y familiares buscando la eficacia manteniendo a los estudiantes más tiempo en las escuelas (Cox, 2012).

Destacaremos tres legislaciones educativas y tres instrumentos orientadores recientes, que impactan en las relaciones entre escuelas y familias. La primera, la Ley de Subvención Escolar Preferencial (SEP) del año 2008, que clasifica a los estudiantes de niveles socioeconómicos bajos como prioritarios (Infante et al., 2013). Con ello se convierten junto a sus familias en fuente de incentivo económico estatal para las escuelas precariamente financiadas (Mizala y Torche, 2012). La segunda, la Ley 20.529, de 2011, crea el Sistema Nacional de Aseguramiento de la Calidad que perfecciona la evaluación mediante indicadores de calidad estandarizados y posibilita que las familias fiscalicen, denunciando cuando las escuelas no cumplen con el servicio que ofertan (Carrasco et al., 2018). La tercera, la Ley 20.845 de Inclusión Escolar del año 2015, prohíbe que los sostenedores privados lucren con los recursos de la subvención estatal destinados a educación y que filtren el ingreso de estudiantes (Sisto, 2019).

En lo que respecta a los tres instrumentos orientadores, el primero es el Decreto 565/1990 que establece funciones diferenciadas para familias y escuelas. Las familias podrán participar organizadamente a través de la instancia formal del Centro de Padres y Apoderados apoyando labores educativas emanadas desde las necesidades de las escuelas, mientras que los aspectos técnico-pedagógicos son de exclusiva competencia de los profesionales de la escuela. El segundo, doce años después, es la Política de Participación de Padres, Madres y Apoderados en el Sistema Educativo. Destaca la importancia de los tutores en la escolarización de su pupilo, sustentada en la cooperación y diversos niveles de participación con el fin de mejorar aprendizajes. Tercero, desde 2017 se encuentra vigente la Política de Participación de las Familias y la Comunidad en Instituciones Educativas, que busca generar condiciones para la participación de las familias y la comunidad en las escuelas, poniendo en el centro el desarrollo integral del estudiantado.

Este marco político influye en los intereses de investigación respecto de las relaciones entre familias y escuelas en el contexto chileno, tal como se presenta en los siguientes apartados. 


\section{3. ¿Cuáles son las conceptualizaciones de familias y escuelas?}

Corresponde preguntarse qué es lo que está siendo investigado en temáticas que construyen como su objeto a las familias y las escuelas de los niños, niñas y adolescentes (NNA). Llama la atención que la literatura revisada rara vez propone definiciones de familias, tampoco las problematiza mayormente. En cambio, se enuncian los cambios sociales y cómo estos afectan a las familias, o los estructurales, sin cuestionar qué compone una familia, qué tipo de relaciones serán consideradas como "familiares" y cómo se diferencia de otras instituciones similares. Se destaca la diversidad de familias, con saberes que han de ser valorados (Julio-Maturana et al., 2016); problematizando el imaginario de una familia idealizada (Precht, 2016, 2018), describiendo los roles que cumplen las familias en la educación de NNA (Gubbins, 2014; Gubbins et al., 2020; Rojas y Falabella, 2015), o el impacto de estas en diferentes tipos de aprendizajes (Saracostti et al., 2019).

Consideramos que, al mencionarse la multiplicidad de ordenamientos familiares sin intentar una definición, se corre el riesgo de continuar invisibilizando dicha diversidad. Atendiendo a los 5.651.637 hogares censales existentes en Chile observamos parte de esta pluralidad. De estos el $19 \%$ es extenso, 2,5\% es compuesto y un $6,6 \%$ son hogares sin núcleos. El $28,8 \%$ son parejas con hijos y el $12,7 \%$ son hogares monoparentales. El $46,6 \%$ corresponde ya sea a hogares sin hijos $12,6 \%$ o es unipersonal 17,8\% (Censo 2017) La noción de hogar solo nos permite acceder a los arreglos domiciliarios de las personas, ignorando si estos pueden coincidir o no con su sentido de pertenencia a una familia.

Es importante señalar que, al investigar sobre escuelas y familias, las definiciones tienden a girar en torno a la función de crianza y su importancia en el desarrollo de los NNA (Ortega y Cárcamo, 2018). En una revisión comparativa de las constituciones chilenas y española respecto del derecho de la familia a participar en la escuela, Castillo y otros (2020) definen familia en tanto núcleo fundamental de la sociedad, el espacio primero de socialización, y al Estado como el encargado de resguardarla -lo mismo en la Constitución chilena, en su Art. $1^{\circ}$ inciso segundo-. Si bien los autores no adhieren a la perspectiva funcionalista, su definición entronca con la tradición parsoniana: un sistema nuclear, unidad más pequeña de parentesco dirigida a la socialización de NNA (Parsons, 1955). Desde otra arista, Razeto (2016) define familia como comunidad de pertenencia que forja la identidad y sostiene vínculos socio afectivos transmitiendo en ella elementos culturales, sociales y espirituales.

En general, se nombra a las familias de un modo genérico, para luego resaltar la importancia de los padres/madres u otros adultos, como los abuelos, que pudieran ser responsables de los estudiantes. Por "familia" se entenderá tácitamente a los adultos de la familia responsables de NNA en relación con su formación escolar. Esta definición de facto trae aparejadas varias dificultades. La primera es su adultocentrismo. Destaca la invisibilización de los NNA que no tienen familiares en condiciones de cuidarles; y aquellos cuya tutela recae en el Estado. Un segundo problema sería la perspectiva alumnizada, NNA son vistos menos desde su pertenencia a una estructura familiar y más desde su posición de estudiantes.

Estos estudios no tienen por foco la familia en sí misma, sino a) la importancia de los tutores de los niños en las resultados del proceso de escolarización y sus aprendizajes (Lara y Saracostti, 2019; Saracostti et al., 2019), las relaciones entre adultos de la comunidad escolar referido a profesionales y tutores (Cárcamo y Garreta, 2020; Castillo et al., 2020; Madrid et al., 2019; Ortega y Cárcamo, 2018; Reininger y Hernández, 2019); y b) las estrategias para preservar el capital social (Canales et al., 2016; Gubbins, 2014;) y cultural asociado a parentocracia (Rojas et al., 2015), así como las consecuencias sociales del neoliberalismo en la comunidad escolar (Precht, 2016; 2018). Estaríamos ante una dificultad de construcción del objeto de estudio, en donde el centro no es "la familia" sino aquella persona adulta que se hace cargo de NNA y su educación ante el sistema educacional.

Algo similar ocurre al problematizar lo escolar; sin embargo, se observa una mayor delimitación del objeto. Distinguimos cuatro escenarios de lo escolar; a) la escuela neoliberal y los cambios que conlleva, escuela cooptada por la lógica gerencialista y la privatización de la vida que transforma la cultura escolar (Precht, 2015a; 2016; 2018; Precht et al., 2016; Saracostti et al., 2019); b) en tanto espacio educativo formal y lugar para el aprendizaje (Razeto, 2018) que complementa la educación familiar (Razeto, 2016); c) como espacio de socialización de NNA con sus pares (Ortega y Cárcamo, 2018) y, finalmente, d) comunidad de aprendizaje en que estudiantes se desarrollan (Ferrada et al., 2014; Miranda y Castillo, 2018; Schilling, 2015). 


\section{4. ¿Para qué se han de relacionar familias y escuelas?}

Independientemente del lugar desde donde se aborde este fenómeno, identificamos un marcador común en torno al para qué de esta relación en la producción científica chilena revisada. Tomando como base el planteamiento aristotélico que sostiene que toda acción humana apunta a la consecución de un bien último, es posible diferenciar respecto de la relación entre familias y escuelas un conjunto de para qué como medios y otro de la relación como un fin.

\subsection{La relación entre familias y escuelas como medio}

No es posible consignar un bloque homogéneo en la relación entre familias y escuelas como un medio. Distinguimos los siguientes para qué: 1) promover la colaboración, 2) generar cambios culturales y, 3) facilitar el desarrollo de capacidades que impacten favorablemente en el desempeño educativo de los NNA.

En cuanto al primero, la relación entre familias y escuelas facilita el desarrollo de la colaboración entre los agentes implicados, tanto para abordar demandas emergentes desde las escuelas, así como problemáticas que afectan a NNA en el transcurso de su escolarización, de naturaleza académica, disciplinar y socioemocional (Castillo et al., 2020; Madrid et al., 2019; Ortega y Cárcamo, 2018; Razeto, 2016, 2018).

El segundo para qué, en tanto medio promueve cambios culturales en el interior de las escuelas. Un tipo de cambio se expresa como posibilidad de modelar espacios dialógicos entre los agentes, superando el nivel informativo prevalente en el modo de hacer escuela (Cárcamo y Garreta, 2020; Cárcamo y Méndez, 2019; Gubbins et al., 2020; Schilling, 2015). Otro cambio destaca el papel de la relación entre familias y escuelas como espacio para la promoción de la formación ciudadana en y desde las propias escuelas (Cárcamo, 2019).

Un tercer para qué como medio busca facilitar el desarrollo de capacidades entre los NNA que transitan por el sistema escolar (Gubbins, 2016; Gubbins y Otero, 2016; Santana y Reininger, 2017; Saracostti et al., 2019). A este respecto, la literatura destaca capacidades cognitivas dirigidas a mejorar los resultados de aprendizaje y también el desarrollo socioemocional que facilita el ajuste del sistema de disposiciones acordes a las demandas del espacio escolar. Conjunto de elementos que redundaría en la disminución de las probabilidades de deserción del sistema educacional.

\subsection{La relación entre familias y escuelas como fin}

A este respecto, los fines pueden ser agrupados en dos grandes dimensiones: académica y sociocultural. Respecto a la dimensión académica, la relación entre familias y escuelas tiene doble finalidad: la mejora del desempeño educativo de NNA verificado a través de la reducción del absentismo (Razeto, 2016, 2018) y la disciplina que tributa al adecuado clima de aula (Razeto, 2018), así como la mejora de los resultados académicos (Gubbins y Otero, 2016).

Referido a la dimensión sociocultural, la relación entre familias y escuelas es entendida como fin en tanto favorece la democratización del espacio escolar. En este sentido, se reconoce el papel que juega el marco normativo vigente a través de la Política de Participación de las Familias y la Comunidad en Instituciones Educativas de 2017; así como también la Ley 20.911 que crea el plan de formación ciudadana un año antes.

Por último, la distinción de relaciones entre familias y escuelas, como medio y fin, conlleva al reconocimiento último -de acuerdo al planteamiento aristotélico- de un bien supremo. De este modo, los autores valoran el vínculo en la medida que conduce a tres fines últimos. El primer fin último se relaciona con la propia vivencia institucionalizada de los NNA en tanto alumnos y radica en mejorar la experiencia de la etapa de escolarización en todos sus niveles (Razeto, 2016, 2018). El segundo, refiere a la vivencia proyectada de los NNA en tanto sujetos adultos; de este modo, la relación entre familias y escuelas tiene como fin último asegurar el futuro de los sujetos escolarizados (Gubbins et al., 2017). Finalmente, el establecimiento de las relaciones entre familias y escuelas conduce a la densificación del tejido social a nivel comunitario (Cárcamo y Méndez, 2019; Ortega y Cárcamo, 2018). 
Cuadro 1

Medios, fines y bien superior de la relación entre familias y escuela

\begin{tabular}{|c|c|c|}
\hline Medio & Fin & Fin último \\
\hline Para promover la colaboración. & $\begin{array}{l}\text { Mejorar la performance educa- } \\
\text { tiva de niños, niñas y adolescen- } \\
\text { tes. }\end{array}$ & $\begin{array}{l}\text { Mejorar la experiencia de la etapa } \\
\text { de escolarización en todos sus } \\
\text { niveles. }\end{array}$ \\
\hline Para generar cambios culturales. & & $\begin{array}{l}\text { Asegurar el futuro de los sujetos } \\
\text { escolarizados. }\end{array}$ \\
\hline $\begin{array}{l}\text { Para facilitar el desarrollo de capa- } \\
\text { cidades que contribuyan a un buen } \\
\text { desempeño académico. }\end{array}$ & $\begin{array}{l}\text { Favorecer la democratización del } \\
\text { espacio escolar. }\end{array}$ & $\begin{array}{l}\text { Densificar el tejido social a nivel } \\
\text { comunitario. }\end{array}$ \\
\hline
\end{tabular}

\section{5. ¿Por qué debe existir esta relación? ¿Cuándo es necesaria?}

Sea para cualquiera de los tres fines últimos, se producen relaciones entre familias y escuelas que parecen responder a las subjetividades contemporáneas. En estas la educación es un servicio ofrecido por la escuela, las escuelas compiten por ofrecer distintos servicios que las familias consumen para sus hijos (Rojas et al., 2015) y para ellas mismos. En este circuito naturalizado las dos instituciones se requieren en momentos específicos por razones puntuales.

Distinguiremos cuatro tipos de razones y momentos que provocan que familias y escuelas se relacionen: las primeras dos son de tipo instrumental motivadas por necesidades de la escuela o de la familia; las segundas son visibles sólo a algunos actores sociales, entre ellos los investigadores: la exigencia de una alianza en pos del estudiante y, las últimas, una preocupación central en las culturas.

El primer tipo de razones y momentos de relaciones es iniciado por las escuelas cuando requieren a las familias para su funcionamiento interno: sea financiero o para el logro de objetivos sociales. Las familias son necesarias para proveer elementos administrativos sin los cuales la escuela no funciona, como los financieros a partir de la subvención estatal entregada contra asistencia diaria de los estudiantes y el financiamiento compartido con las familias. Por lo tanto, un momento de relación es cuando los NNA no asisten a la escuela, provocando el despliegue de diversas acciones de comunicación con las familias, como las visitas domiciliarias (Razeto, 2018). También se relacionan para solicitar la firma de los apoderados para autorizaciones diversas (paseos, vacunas, etc.) o para solicitar elementos materiales como uniformes escolares, listas de útiles o requerimientos para las actividades habituales.

Las metas sociales son otra razón para que escuelas y familias se relacionen. Los servicios educativos se valoran desde la sociedad en función de su excelencia académica, la cual es destacada en rankings y viene acompañada de prestigio social. El supuesto en la base es que los agentes escolares deben esforzarse: profesores, estudiantes y familias deben destacar por sus resultados en mediciones estandarizadas. En este sentido se asume que la participación de las familias en la escuela hace la diferencia para alcanzar el éxito escolar (Gubbins et al., 2020; Miranda y Castillo, 2018) dependiendo en gran medida de la presencia/ausencia familiar. Esta es una creencia compartida por profesores en ejercicio (Julio-Maturana et al., 2016) como por aquellos en formación (Cárcamo y Garreta, 2020; Precht et al., 2016), ya que participación familiar y desarrollo socioemocional serían predictores de los resultados del aprendizaje (Saracostti et al., 2019).

El segundo tipo de razones y momentos, también funcionales, surge de una actitud proactiva de las familias, las cuales desconfían de las escuelas: como la educación entregada por las escuelas es insuficiente, se asume un rol familiar activo, estratégico y consciente (Rojas et al., 2015). La sociedad indica que la educación es imprescindible para la movilidad o conservación del estatus social y económico (Canales et al., 2016) y, dado que las escuelas no lo aseguran, es necesario que las familias actúen: esforzándose por estar presentes en la escuela, lo que Rojas y otros (2015) denominan parentocracia, o utilizando los incentivos jurídicos para supervisarla (Castillo et al., 2020).

Un tercer grupo de razones y momentos, amparado en los lineamientos de política pública, plantea compartir la responsabilidad de educar a NNA. Ambas instituciones ponen al centro al NNA en tanto estu- 
diante y colaboran recíprocamente (Castillo et al., 2020) abordando las problemáticas que conjuntamente atraviesan los estudiantes. Tal como lo denunció Baquedano (2013) respecto de Estados Unidos, las políticas públicas suelen apoyarse en la propuesta de involucramiento parental de Epstein (1992, 2010) y de Epstein y Hollifield (1996) con su teoría de las esferas de influencia (escuela, familia y comunidad) aliadas en metas, misiones y responsabilidades comunes. La Política de participación de las familias y la comunidad en instituciones educativas está fuertemente influida por Epstein justificando la necesidad de una alianza entre escuelas y familias. La teoría de Epstein supone sin embargo una igual capacidad de agencia por parte de madres, padres y tutores para plantear y defender sus posiciones, desconociendo asimetrías de poder. Dicha "alianza" olvida las desigualdades e intersecciones posibles de género, clase, etnia u origen geográfico (local o migrante) y asume que todos los miembros de la comunidad escolar intencionan y acogen los mismos propósitos educativos. Un riesgo de este enfoque es comprender cualquier desviación del involucramiento como un déficit de parentalidad (Baquedano et al., 2013). La metáfora de la "alianza" es funcional a perspectivas que promuevan el individualismo, dado que se centra en las demandas que las escuelas realizan a las familias y a una relación que intenciona vínculos clientelares.

Finalmente, un cuarto grupo de razones, ocurre cuando la sociedad concibe a las familias como fuente valiosa de saberes. Esto no necesariamente dialoga con el currículo formal oficial y occidentalizado (Quilaqueo et al., 2014), quedando más bien reducido a las familias poseedoras de un currículo propio (Julio-Maturana et al., 2016). La relación se produce a partir de problemáticas investigativas construidas y desarrolladas con la comunidad escolar, en sus propios contextos, con la finalidad consensuada de impulsar procesos de transformación (Ferrada et al., 2014). El abordaje antropológico e intercultural evidencia el desencuentro cultural en las escuelas cuando los saberes familiares no son valorados (Julio-Maturana et al., 2016) y el reduccionismo curricular deslegitima los saberes situados, al homogeneizar la cultura.

Estas razones que buscan vincular a las escuelas y familias incluyen la valoración de saberes culturales, por una parte, de pueblos originarios como el mapuche (Quilaqueo et al., 2014), de grupos excluidos (Julio-Maturana et al., 2016), así como la activación de comunidades que, desde un enfoque territorial, involucran a los actores sociales que se suman a la educación (Ferrada et al., 2014). Asimismo, problematizan las formas de conocer y el qué se conoce, apropiándose del currículo deseado por su comunidad escolar. Así, esta relación dialógica incluye a las familias y comunidad del entorno no solo en las instancias curriculares escolares, sino que en todos los espacios en que se piensa la educación (Ferrada et al., 2014).

\section{6. ¿Cómo se concibe la relación entre familias y escuelas?}

El cómo se entiende la relación entre familias y escuelas, corresponde a la interpretación de la referencia a familias y a escuelas en los estudios analizados en el contexto chileno, los cuales pueden ser agrupados en tres lógicas:

\subsection{Lógica de mercado educativo}

Los estudios de Gubbins $(2014,2016)$ se enfocan en cómo la relación entre familias y escuelas en Chile se enmarca en una lógica de consumo de servicios educacionales, que tiene por objetivo vender y comprar un bien. En este caso, educación para los sujetos en edad escolar por parte de las familias, enmarcada en la condición de cliente de la familia según su condición social y elección del servicio educativo para sus NNA: son ellas quienes pagan por un servicio. Rojas y otros (2015) comprende dicha relación en tanto mediada por las directrices de las políticas públicas y sujeta a ellas, donde las familias asumen el rol de "parentalidad responsable" para asegurar el futuro de sus hijos. Esto se traduce en la gestión de ciertos "riesgos sociales" que influyen al momento de elegir el establecimiento educacional para sus hijos (Rojas, 2018). En Precht $(2016,2018)$ y Precht y otros (2016) esta relación se entiende enmarcada en las políticas neoliberales y elementos estructurales que las posibilitan.

Entre los estudios que analizan esta lógica del consumo educativo neoliberal se distinguen aquellos que enfocan la relación entre familias y escuelas orientada al aseguramiento del futuro -en términos académicos- y de reproducción de privilegios (Gubbins, 2014): se trata de un proyecto familiar de futuro del que pretenden formar parte (Rojas et al., 2015). Mientras, otros entienden dicha relación como el aseguramiento que gestionan los padres frente a la sociedad del riesgo que se percibe en la actualidad. Es el 
caso de estudios centrados en familias de clase media baja (Canales et al., 2016; Ramos, 2017) donde la relación entre familias y escuelas está orientada no tanto por la calidad de los servicios educativos sino por el buen ambiente (de seguridad) que la escuela provee.

\subsection{Lógica de oportunidad de mejoramiento}

Esta forma de relación se basa en estudios que centran su mirada en el proceso de escolarización. Así, Razeto (2016) ubica la relación como un deber con propósitos de mejoramiento. Este deber responsabiliza a las familias a involucrarse en tiempos y espacios institucionales escolares y otras veces a las escuelas (Razeto, 2018), principalmente a equipos directivos, de promover la participación de las familias. Del mismo modo, Castillo y otros (2020) entienden la relación como un deber ser que trasciende la búsqueda de la eficiencia educativa, hacia una conducta cooperativa que implica involucramiento familiar colaborativo, entendido como la "disposición de la familia a participar y vincularse a la escuela y los aprendizajes de los hijos, incluyendo las prácticas conductuales y verbales en el hogar, así como las que se despliegan en las actividades que organiza la escuela" (p. 27). Familias y escuelas serían "igualmente protagonistas" y se debe buscar el empoderamiento de ambas (Madrid et al., 2019).

En Precht y otros (2016) se comprende esta relación como una necesidad para el mantenimiento del esfuerzo en la tarea de educar que realiza la escuela. En contextos rurales, la relación sería facilitadora en el proceso de convertir al niño en estudiante, lo que se traduce en que la escuela llama a la participación de estas madres campesinas para finalidades logísticas de aseo, cocina o elaboración de disfraces, en una reproducción de su posición social (Precht, 2015b).

En los estudios de Cárcamo y colaboradores, se entiende la relación entre familias y escuelas como una alianza con propósitos en dos niveles: hacia el aprendizaje y calidad educativa, y hacia la democratización del espacio escolar. La construcción de espacios dialógicos en una interacción entre familias y escuelas en todos los momentos formales e informales del proceso educativo (Cárcamo y Méndez, 2019; Ortega y Cárcamo, 2018) permitiría entender que familias y escuelas tienen voz y reconocimiento mutuo.

\subsection{Lógica de reconocimiento de las culturas familiares}

La última forma de relación se basa en el reconocimiento de los aspectos culturales y significaciones asumiendo la perspectiva de Geertz (2005). Julio-Maturana y otros (2016) reconoce la construcción identitaria y cultural de los NNA en sus familias y currículum familiar, quienes siempre se desarrollan acompañados de adultos y en un entorno cultural (Ferrada et al., 2014).

Desde una perspectiva intercultural, Quilaqueo y otros (2014) destacan la relevancia de los contenidos indígenas de la cultura Mapuche que forman parte de la educación familiar. Se observa que el desconocimiento de los saberes educativos mapuches dificulta la implementación de un enfoque educativo más adaptado a los conocimientos propios de su cultura, en el caso de los alumnos de origen tanto mapuche como no mapuche. Por una parte, los autores entienden necesaria la relación entre familias y escuelas, al incluir el conocimiento contextualizado, en tiempo y espacio, procedencia geográfica, ascendencia parental. Asimismo, es la propia familia y comunidad la que educa en estos saberes. De esta manera, apuestan por una educación contextualizada que potencie la formación de los NNA a partir del diálogo de saberes desde un enfoque intercultural.

Un aporte decolonialista es el de la pedagogía comunitaria (Schilling, 2011) en el marco de la investigación dialógica-kishu kimkelay ta che en educación, el cual rescata la reciprocidad gnoseológica entre los integrantes de la comunidad escolar con procedimientos de producción de conocimiento colectivo, permitiendo que toda la comunidad aprenda (Ferrada et al., 2014).

Así, la educación intercultural como propuesta epistémica tiende a reducir la asimetría que existe acerca del conocimiento familiar por parte de las escuelas, y de la misma manera, la asimetría en las relaciones entre ambas instituciones. 


\section{A modo de cierre}

Poner bajo una lupa a las investigaciones chilenas que involucran a familias y escuelas como objeto de investigación, nos entrega información valiosa acerca de la manera en que cada uno de nosotros como investigadores alimenta los discursos sobre estas dos instituciones en el ámbito educativo. No solemos incluir una definición clara de familia en los escritos, pese a que es parte del objeto de estudio. Desde una actitud de vigilancia epistémica, se destacan dos ideas: la forma de configurar la noción de familia y, las maneras en que familias y escuelas acuden entre sí.

Respecto a las formas de configurar la familia, hay un claro desbalance entre la precisión con la que se define escuela y la imprecisión respecto de familia. Pareciera que los investigadores optamos por hablar de las familias desde los resultados de investigación, evitando una definición inicial. El término familia es utilizado en los estudios indistintamente, sin distinguir cuando nos referimos a adultos responsables del estudiante (madre, padre, abuelos, tutores u otros) o a su familia como colectivo, lo cual produce efectos sobre sus configuraciones actuales, responsabilizando a unos e invisibilizando a otros respecto del quehacer de educar. Además, se produce un problema epistémico en las formas de comunicar lo que es una familia, primando lo que otros dicen sin declarar un posicionamiento de la investigación y, por lo tanto, de sus investigadores.

En cuanto a las maneras en que ambas instituciones acuden una a la otra, destaca que la relación es instrumental. Se asume que miembros de ambas instituciones están dispuestos a establecer las relaciones de colaboración, comunicación, participación, alianza, etc., como reglas del juego establecidas desde las escuelas, o más bien por las políticas públicas neoliberales.

Vemos una oportunidad en este ejercicio de reflexión: tanto para la formación de profesores como para la investigación. En cuanto a la formación inicial docente, planteamos la importancia de transparentar fines, razones y maneras de significar relaciones entre familias y escuelas. Por una parte, es necesario abordar la pluralidad de familias y sus necesidades más allá de las metas administrativas y de logro social de las escuelas. Por otra parte, es urgente desnaturalizar que siempre hay una familia presente para disminuir el riesgo social al que se exponen NNA. El futuro de NNA y su educación escolar no puede basarse en el requisito familiar; esto cuestiona el enfoque de derecho a la educación. ¿Y si ese estudiante no tiene una familia que pueda entrar en la relación exigida?

En cuanto a la investigación, este ejercicio nos desafía a mantener, por un lado, una vigilancia epistemológica frente a los riesgos de caer en una lógica neoliberal que homogeniza tanto a familias como a escuelas, exigiendo responsabilizar y cooperar en pos de la calidad y la eficacia. Esta mirada reduccionista adjetiva el rol de las familias de antemano en la deslegitimación, estrechando su definición a familia escolarizada. Por otro lado, nos pide a los investigadores un posicionamiento más activo, destacando las dimensiones gnoseológica y epistemológica. Tal como la teoría de los Fondos de Conocimiento (Moll et al., 2001, Moll, 2014, Amanti, 2020), o la de las aulas dialógicas de Ferrada et al. (2014), quienes dan cuenta de la necesidad urgente de un doble giro: desde lo individual e instrumental, hacia lo comunitario y lo dialógico.

La naturalización de valores neoliberales, como el individualismo, la competencia, el consumismo, el lucro nos llama como investigadores más allá de la resistencia a una revaloración de lo comunitario y resignificación de las relaciones tradicionalmente establecidas. La comunidad escolar puede dar soporte al desarrollo de los NNA que crecen rodeados de adultos (Ferrada et al., 2014), lo que no está exento de conflicto dentro de esa organización, e incluso las relaciones de poder son parte de lo que la escuela hace $y$, el disenso y la diversidad de opiniones e intereses debe reconocerse dentro de la comunidad.

Más que una relación funcional, se trata de que los estudios expliciten el fin último democrático que los orienta, colaborando en que escuelas y familias se legitimen recíprocamente mediante el diálogo. Más que rigidizar aún más las estructuras, las investigaciones pueden favorecer otras formas de entender las relaciones y de marcar los límites y funciones entre sus miembros. Esto nos devuelve a un punto de partida antropológico y a una mirada sistémica más compleja.

La investigación, como quehacer social, tiene un efecto performativo y puede sustentar la naturalización de las familias funcionales/disfuncionales y su consecuente estigmatización, reproduciendo esta indefinición e invisibilizando la diversidad de familias y los cambios culturales recientes que han llevado a 
transformarlas. Sospechar sobre la noción de familia cristalizada, más aún en el momento histórico, social y político de Chile, nos invita a explicitar la noción de familias basada en la experiencia subjetiva y dialógica de las comunidades educativas y, a recuperar la naturaleza intercultural de las relaciones entre familias y escuelas.

\section{Agradecimientos}

Esta investigación ha sido financiada por la Asociación Nacional de Investigación y Desarrollo (ANID) a través del Proyecto Fondecyt Regular 1181925 y el programa ANID-PIA CIE 160009.

Los autores afirman que no hay conflicto de intereses en este artículo, y todos ellos han contribuido por igual en el artículo.

En memoria de Paulina Carrasco Delgado, miembro del equipo de investigación.

\section{Referencias}

Alarcón, J. y Donoso, S. (2017). Hitos significativos de la política educacional del gobierno de la coalición por el cambio (2010-2014). Estudios Pedagógicos, 43(1), 371-388. https://doi.org/10.4067/S0718-07052017000100021

Amanti, C. (2020). Desafiando perspectivas deficitarias acerca de estudiantes con origen obrero como minorías. En López, V. (Ed.), Fondos de conocimientos: propuestas para trabajar desde las sabidurías de las familias (pp. 4-13). Centro de Investigación para la Educación Inclusiva.

Angulo, F. (2019). Evaluación y estándares en educación: legitimidad y equidad. EduInclusiva.

Baquedano-López, P., Alexander, R. A. and Hernández, S. J. (2013). Equity issues in parental and community involvement in schools what teacher educators need to know. Review of Research in Education, 37(1), 149-182. https://doi.org/10.3102/0091732X12459718

Bellei, C., Contreras, D. y Valenzuela, J. (Eds.). (2008). La agenda pendiente en educación. PNUD.

Canales, M., Bellei, C. y Orellana, V. (2016). ¿Por qué elegir una escuela particular subvencionada? Sectores medios emergentes y elección de escuela en un sistema de mercado. Estudios Pedagógicos, 42(3), 89-109. https://doi.org/10.4067/S0718-07052016000400005

Cárcamo, H. y Garreta, J. (2020). Representaciones sociales de la relación familia-escuela desde la formación inicial del profesorado. Revista Electrónica de Investigación Educativa, 22, 1-14.

https://doi.org/10.24320/redie.2020.22.e11.2406

Cárcamo, H. y Méndez, P. (2019). La influencia de la experiencia en el modelamiento de las representaciones sociales sobre la relación familia-escuela en establecimientos educacionales rurales de la región de Ñuble, Chile. Revista Electrónica Educare, 23(3), 1-20. https://doi.org/10.15359/ree.23-3.4

Cárcamo, H. (2019). Relación familias-escuela y procesos de formación ciudadana. En G. Orellana, M. Salazar y H. Hasse (Eds.), Formación ciudadana en el contexto escolar. Conceptualización, avancesy experiencias (pp. 29-46). RIL. https://doi.org/10.20511/pyr2020.v8n2.408

Carrasco, C., Acorra, P., López, V. y Álvarez, J. (2018). Tensiones normativas de los fiscalizadores de la Superintendencia de Educación en la(s) política(s) de convivencia escolar en Chile. Perfiles Educativos, 40(159), 126-143. https://doi.org/10.22201/iisue.24486167e.2018.159.58153

Castillo, L., Saracostti, M., Castellanos, J. y Morales, S. (2020). Derecho de las familias a participar en el contexto escolar: análisis desde las constituciones chilena y española. Revista Jurídicas, 17(1), 125-141.

Cerletti, A. (2008). La enseñanza de la filosofía como problema filosófico. Zorzal.

Cox, C. (2012). Política y políticas educacionales en Chile 1990-2010. Revista Uruguaya de Ciencia Política, 21(1), 13-42.

Díaz de Rada, A. (2010). Cultura, antropología y otras tonterías. Trotta. 
Donoso-Díaz, S., Souza, A. y Barbosa Gouveia, A. (2016). Desafíos políticos claves para los sistemas escolares de Brasil y Chile. Revista Española de Educación Comparada, 27, 73-96. https://doi.org/10.5944/reec.27.2016.16429

Epstein, J. L. (1992). School and family partnerships. Encyclopedia of educational research. MacMillan.

Epstein, J. L. (2010). School/family/community partnerships: caring for the children we share. Phi Delta Kappan, 92(3), 81-96. https://doi.org/10.1177/003172171009200326

Epstein, J. L. and Hollifield, J. H. (1996). Title I and school-family-community partnerships: using research to realize the potential. Journal of Education for Students Placed at Risk, 1(3), 263-278.

https://doi.org/10.1207/s15327671espr0103_6

Esteban-Guitart, M., Oller, J., y Vila, I. (2012). Vinculando escuela, familia y comunidad a través de los fondos de conocimiento e identidad. Un estudio de caso con una familia de origen marroquí. Revista de Investigación en Educación, 10(2), 21-34. http://reined.webs.uvigo.es/index.php/reined/article/view/148

Ferrada, D., Villena, A., Catriquir, D., Pozo, G., Turra, O., Schilling, C. y Del Pino, M. (2014). Investigación dialógica-kishu kimkelay ta che en educación. REXE. Revista de Estudios y Experiencias en Educación, 13(26), 33-50.

Geertz, C. (2005). La interpretación de las culturas. Gedisa.

Gubbins, F. V. (2014). Estrategias educativas de familias de clase alta: Un estudio exploratorio. Revista mexicana de investigación educativa, 19(63), 1069-1089.

Gubbins, V. (2016). Relación familias y escuelas: ¿por qué y para qué? Ediciones Finis Terrae.

Gubbins, V. and Otero, G. (2016). Effects of chilean schools' accountability on parent participation. Revista de Educación, 372, 9-34. https://doi.org/10.4438/1988-592X-RE-2015-372-313

Gubbins, V., Ugarte, E. y Cárcamo, H. (2020). Estilos comunicativos docentes y su incidencia en los modos de participación de los padres desde la mirada de madres de grupos vulnerables. Revista Propósitos y Representaciones, 8(2), e408. https://doi.org/10.20511/pyr2020.v8n2.408

Gubbins, V., Tirado, V. y Marchant, V. (2017). Disposiciones docentes hacia la relación con apoderados en un establecimiento educacional privado en Chile: estudio de caso. Perfiles Educativos, 39(155), 107-122. https://doi.org/10.22201/iisue.24486167e.2017.155.58109

Infante, M., Matus, C., Paulsen, A., Salazar, A. y Vizcarra, R. (2013). Narrando la vulnerabilidad escolar: Perfomatividad, espacio y territorio. Literatura y Lingüistica, 27, 281-308. https://scielo.conicyt.cl/pdf/lyl/n27/art14.pdf

Julio-Maturana, C., Conejeros-Solar, L., Rojas, C., Mohammad, M., Rubí, Y. y Cortés, Y. (2016). Desencuentro cultural en el aula: una barrera al aprendizaje de niños y niñas en situación de pobreza. RMIE, 21(68), 71-94.

Lara, L. y Saracostti, M. (2019). Effect of parental involvement on children's academic achievement in Chile. Frontiers in Psychology, 10, 1464. https://doi.org/10.3389/fpsyg.2019.01464

Madrid, R., Saracostti, M., Reininger, T. y Hernández, M. T. (2019). Responsabilización, obediencia y resistencia: perspectivas de docentes y padres sobre la colaboración familia-escuela. Revista Electrónica Interuniversitaria de Formación del Profesorado, 22(3), 1-13. https://doi.org/10.6018/reifop.389801

Manghi, D., Conejeros, M., Bustos, A., Aranda, I., Vega, V. y Díaz, K. (2020). Comprender la educación inclusiva chilena: panorama de políticas e investigación educativa. Cadernos de Pesquisa, 50(175), 114-135.

https://doi.org/10.1590/198053146605

Miranda, C. y Castillo, P. (2018). Estudio metodológico de las variables que componen el constructo de apoyo y participación de la familia en los procesos educativos. Estudios Pedagógicos, 44(1), 115-133. https://doi.org/10.4067/S0718-07052018000100115

Mizala, A. y Torche, F. (2012). Bringing the schools back in: The stratification of educational achievement in the Chilean voucher system. International Journal of Educational Development, 32(1), 132-144. https://doi.org/10.1016/j.ijedudev.2010.09.004

Moll, L. (2014). Vygotsky and education. Routledge. https://doi.org/10.4324/9780203156773 
Moll, L., Amanti, C., Neff, D. y Gonzalez, N. (2001). Funds of knowledge for teaching: using a qualitative approach to connect homes and classrooms. Theory into Practice, 31(2), 132-141. https://www.jstor.org/stable/1476399

Ortega, M. y Cárcamo, H. (2018). Relación familia-escuela en el contexto rural. Miradas desde las familias. Educación, 52, 98-118. https://doi.org/10.18800/educacion.201801.006

Parsons, T. (1955). The american family: its relations to personality and to the social structure. En T. Parsons y R. Bales (Eds.), Family. Socialization and interaction process. Glencoe, Ill: The Free Press.

Precht, A. (2015a). ¿Qué le duele a la escuela? Problematizando el malestar de docentes y apoderados. En V. Nogués y A. Precht (Eds.), Nuevas formas de relación en la escuela: reflexionar y transformar (pp. 19-28.). Ediciones Universidad Santo Tomás.

Precht, A. (2015b). Rural parents perceptions about parents meetings. The Turkish Online Journal of Educational Technology, 9, 439-445.

Precht, A. (2016). Privatización de la relación entre familias y escuela: Un aspecto a considerar en la convivencia escolar. En G. Salas (Ed.), Del pathos al ethos: líneas y perspectivas de la convivencia escolar (pp. 129-142). Ediciones UCM.

Precht, A. (2018). Nos vienen a tirar a sus hijos a la escuela: Problematizando la relación entre familia y escuela en tiempos de intensificación de la parentalidad. En D. Ferrada (Ed.), Reflexiones y experiencias educativas desde las comunidades. Investigación en educación para la justicia social (pp. 119-132). Ediciones UCM.

Precht, A., Valenzuela, J., Muñoz, C. y Sepúlveda, K. (2016). Familia y motivación escolar: Desafíos para la formación inicial docente. Estudios Pedagógicos, 42(4), 165-182. https://doi.org/10.4067/S0718-07052016000500010

Quilaqueo, D., Quintriqueo, S., Torres, H. y Muñoz, G. (2014). Saberes educativos mapuches: Aportes epistémicos para un enfoque de educación intercultural. Chungara, Revista de Antropología Chilena, 46(2), 271-283. https://doi.org/10.4067/S0717-73562014000200008

Ramos, M. (2017). Hows and whys of parental involvement in a national neoliberal laboratory: aspirations, values and beliefs in relation to children's education among Chilean urban lower-middle-class parents. Compare: $A$ Journal of Comparative and International Education, 47(6), 925-941. https://doi.org/10.1080/03057925.2017.1375849

Razeto, A. (2016). El involucramiento de las familias en la educación de los niños. Cuatro reflexiones para fortalecer la relación entre familias y escuelas. Páginas de Educación, 9(2), 190-216. https://doi.org/10.22235/pe.v9i2.1298

Razeto, A. (2018). Estrategias para promover la participación de familias en la educación de niños en escuelas chilenas. Revista Educacao e Pesquisa, 44, e180495. 1-20. https://doi.org/10.1590/S1678-4634201844180495

Reininger, T. y Hernández, M. T. (2019). Responsabilización, obediencia y resistencia: Perspectivas de docentes y padres sobre la colaboración familia-escuela. Revista Electrónica Interuniversitaria de Formación del Profesorado, 22(3), 1-13. https://doi.org/10.6018/reifop.389801

Rojas, M. (2018). Inclusión social: Miradas de los docentes y apoderados frente a la mixtura social en sus escuelas. Estudios Pedagógicos, 44(3), 237-254. https://doi.org/10.4067/S0718-07052018000300217

Rojas, M., Falabella, A. y Leyton, D. (2015). Madres de clases medias frente al mercado educativo en Chile: Decisiones y dilemas. En J. Corvalán (Eds.), Mercado escolary oportunidad educacional: Libertad, diversidad y desigualdad (pp. 233-268). Ediciones UCE. https://doi.org/10.2307/j.ctv14rmrhn.11

Santana, A. y Reininger, T. (2017). El involucramiento familiar en el marco de la relación familia-escuela. Un análisis desde el equipo de gestión escolar en una escuela municipal chilena. Comunitaria Revista Internacional de Trabajo Social y Ciencias Sociales, 14, 55-74.

Saracostti, M., Lara, L., Martella, D., Miranda, H., Miranda-Zapata, E. D. y Reininger, T. (2019). Influence of family involvement and children's socioemotional development on the learning outcomes of Chilean students. Frontiers in Psychology, 10, 335, 1-7. https://doi.org/10.3389/fpsyg.2019.00335

Schilling, C. (2011). La práctica pedagógica comunitaria: Una experiencia de reconstrucción dialógica y colaborativa desde el contexto de comunidades que viven la adversidad. REXE, 10(20), 75-90. 
Schilling, C. (2015). La construcción de nuevas interacciones entre familia y escuela: avanzando hacia una perspectiva comunicativa del espacio escolar. En V. Nogués y A. Precht (Eds.), Nuevas formas de relación en la escuela: Reflexionar y transformar (pp. 195-214). Universidad Santo Tomás.

Sisto, V. (2019). Inclusión "a la chilena”: La inclusión escolar en un contexto de políticas neoliberales avanzadas. Archivos Analíticos de Políticas Educativas, Arizona, 27(23), 1-20. https://doi.org/10.14507/epaa.27.3044

Slee, R. y Allan, J. (2001). Excluding the included: A recognition of inclusive education. International Studies in Sociology of Education, 11(2), 173-191. https://doi.org/10.1080/09620210100200073

Vélez-Ibáñez, C. y Greenberg, J. (2005). Formation and transformation of funds of knowledge. En N. González (Ed.), Funds of knowledge: Theorizing practices in households, communities, and classrooms (pp. 47-70). Routledge.

Zemelman, H. (2005). Voluntad de conocer. El sujeto y su pensamiento en el paradigma crítico. Anthropos.

\section{Breve CV de los/as autores/as}

\section{Dominique Manghi}

Doctora en Lingüística y Licenciada en Educación. Actualmente es profesora adjunta de la Escuela de Pedagogía de la Pontificia Universidad Católica de Valparaíso e investigadora principal de la Línea 4 Prácticas de aula y escuela para la inclusión del Centro de Investigación para la Educación Inclusiva. Forma profesoras/es y participa en diversos proyectos y equipos de investigación. Las áreas de interés se encuentran en el ámbito educativo y discursivo, investigando en prácticas educativas, interacción en espacios escolares, mirada sistémica a la educación, discursos de actores educativos y discurso educativo en las redes. Su enfoque principal es desde la semiótica social y la multimodalidad.

Email: dominique.manghi@pucv.cl

ORCID ID: https://orcid.org/0000-0002-0278-9899

\section{Elsa María Castrillón-Correa}

Candidata a Doctora en Educación del programa de Doctorado en Educación en Consorcio de la Universidad Católica de Maule (Chile). Profesional de Desarrollo Familiar de la Universidad de Caldas y Máster en Desarrollo Sostenible y Medio Ambiente de la Universidad de Manizales, ambos en Colombia. Participa en el Proyecto Fondecyt Regular $\mathrm{N}^{\circ} 1181925$ sobre las formas de Agenciamiento de madres en la educación de escolares. El interés de investigación se centra en las relaciones entre familia escuela, la agencia humana y sus relaciones con los individuos, así como en contextos colectivos como la familia y la escuela. El enfoque de investigación es interpretativo-crítico. Email: elsa.castrillon@alu.ucm.cl

ORCID ID: https://orcid.org/0000-0001-7340-2287

\section{Héctor Cárcamo-Vásquez}

Sociólogo, Licenciado en Sociología y Magíster en Investigación Social de la Universidad de Concepción, Chile. Doctor en Antropología Social de la Universidad Complutense de Madrid, España. Está adscrito al Departamento de Ciencias Sociales de la Universidad del Bío-Bío, Chile. Es investigador responsable del Grupo de Investigación Familia, Escuela y Sociedad (FESOC), financiado por la Dirección de Investigación y Creación Artística de la Universidad del Bío-Bío. Las líneas de investigación desarrolladas corresponden, por una parte, a la relación entre familias y escuelas y, por otra, a la ciudadanía y procesos de formación ciudadana en contextos educativos formales. Email: hcarcamo@ubiobio.cl

ORCID ID: https://orcid.org/0000-0003-2045-343X

\section{Andrea Precht}

Profesora adscrita al Departamento de Fundamentos de la Educación en la Facultad de Ciencias de la Educación de la Universidad Católica del Maule (Chile). Es investigadora asociada al Centro de Investi- 
gación en Educación para la Justicia Social (CIEJUS) de la misma universidad. Es Doctora en Ciencias de la Educación por la Pontificia Universidad Católica del Maule. Actualmente es miembro de los Claustros académicos del Doctorado en Educación y Doctorado en Psicología (UCM). Sus líneas de investigación son: Familias, parentalidades y escuelas; así como, formación de profesores y procesos motivacionales en Educación Superior. Email: aprecht@ucm.cl

ORCID ID: https://orcid.org/0000-0002-2435-5565 